\title{
The SST-1M project for the Cherenkov Telescope Array
}

M. Heller ${ }^{* a}$, , C. Alispach ${ }^{a}$, I. Al Samarai ${ }^{a}$, M. Balbo ${ }^{b}$, A. Barbano ${ }^{a}$, V. Beshley ${ }^{o}$, A. Biland ${ }^{c}$, J. Blazek ${ }^{i}$, J. Błocki ${ }^{d}$, J. Borkowski ${ }^{h}$, T. Bulik ${ }^{e}$, F. Cadoux ${ }^{a}$, L. Chytka ${ }^{l}$, V. Coco ${ }^{a}$, N. De Angelis ${ }^{a}$, D. della Volpe ${ }^{a}$, Y. Favre ${ }^{a}$, T. Gieras ${ }^{d}$, M. Grudzińska ${ }^{e}$, P. Hamal ${ }^{l}$, M. Hrabovsky ${ }^{l}$, J. Juryšek ${ }^{i, l}$, J. Kasperek ${ }^{k}$, K. Koncewicz $^{d}$, A. Kotarba ${ }^{d}$, E. Lyard ${ }^{b}$, E. Mach ${ }^{d}$, D. Mandat ${ }^{i}$, S. Michall ${ }^{l}$ J. Michałowski ${ }^{d}$, R. Moderski ${ }^{h}$, T. Montaruli ${ }^{a}$, A. Nagai ${ }^{a}$, D. Neise ${ }^{c}$, J. Niemiec ${ }^{d}$, T.R.S. Njoh Ekoume ${ }^{a}$, M. Ostrowski ${ }^{f}$, M. Palatka ${ }^{i}$, P. Paśko ${ }^{g}$, M. Pech $^{i}$, B. Pilszyk ${ }^{d}$, H. Przybilski ${ }^{d}$, P. Rajda $^{k}$, Y. Renier ${ }^{a}$, P. Rozwadowski ${ }^{e}$, P. Schovanek ${ }^{i}$, K. Seweryn ${ }^{g}$, V. Sliusar ${ }^{b}$, D. Smakulska ${ }^{d}$, D. Sobczyńska ${ }^{n}$, Ł. Stawarz ${ }^{f}$, J. Świerblewski $^{d}$, P. Świerk $^{d}$, P. Travnicek ${ }^{i}$, I. Troyano Pujadas ${ }^{a}$, R. Walter ${ }^{b}$, M. Wiecek $^{d}$, A. Zagdański ${ }^{f}$, K. Ziętara ${ }^{f}$, for the CTA-SST-1M project

${ }^{a}$ DPNC - Université de Genève, 24 Quai Ernest Ansermet, CH-1211 Genève, Switzerland

${ }^{b}$ Département d'Astronomie, Université de Genève, Chemin d'Ecogia 16, CH-1290 Versoix, Switzerland

${ }^{c}$ ETH Zurich, Institute for Particle Physics and Astrophysics, Otto-Stern-Weg 5, 8093 Zurich, Switzerland

${ }^{d}$ Institute of Nuclear Physics Polish Academy of Sciences, PL-31342 Krakow, Poland

${ }^{e}$ Astronomical Observatory, University of Warsaw, Al. Ujazdowskie 4, 00-478 Warsaw, Poland

${ }^{f}$ Astronomical Observatory, Jagiellonian University, ul. Orla 171, 30-244 Kraków, Poland

${ }^{g}$ Centrum Badań Kosmicznych Polskiej Akademii Nauk, 18 a Bartycka str., 00-716 Warsaw, Poland

${ }^{h}$ Nicolaus Copernicus Astronomical Center, Polish Academy of Sciences, ul. Bartycka 18, 00-716 Warsaw, Poland

${ }^{i}$ FZU - Institute of Physics of the Czech Academy of Sciences, 17. listopadu 50, Olomouc \& Na Slovance 2, Prague, Czech Republic.

${ }^{k}$ AGH University of Science and Technology, al.Mickiewicza 30, 30-059 Kraków, Poland

${ }^{l}$ Palacky University Olomouc, Faculty of Science, RCPTM, 17. listopadu 50, Olomouc, Czech Republic.

${ }^{n}$ Department of Astrophysics, University of Łódź, ul. Pomorska 149/153, 90-236 Łódź, Poland

${ }^{\circ}$ Pidstryhach Institute for Applied Problems of Mechanics and Mathematics, National Academy of Sciences of Ukraine, 3-b Naukova St., 79060, Lviv, Ukraine

E-mail: matthieu.hellereunige.ch

The SST-1M project, run by a Consortium of institutes from Czech Republic, Poland and Switzerland, has been proposed as a solution for implementing the small-size telescope array of the southern site of the Cherenkov Telescope Array. The technology is a pathfinder for efficient production of cost-effective imaging air Cherenkov telescopes. We report on the main system features and recent upgrades, the performances validation and the operation campaign carried out in 2018 .

36th International Cosmic Ray Conference -ICRC2019-

July 24th - August 1st, 2019

Madison, WI, U.S.A. 


\section{Introduction}

The Cherenkov Telescope Array (CTA) is the future very-high energy gamma-ray astronomical observatory. It is composed of a Southern and a Northern array of groundbased telescopes for measuring gamma rays from about $20 \mathrm{GeV}$ to $300 \mathrm{TeV}$. A sub-array of CTA will be composed of Small Size Telescopes (SSTs) and will be dedicated to the high energy region of the gamma spectrum, from 3 to $300 \mathrm{TeV}$. In order to acquire enough statistics at such high energies, the SST sub-array will need to cover a large (several $\mathrm{km}^{2}$ ) surface on the ground. Key features for the prototyping of the SST telescopes are the low cost and the large scale producibility. Fig. 1 summarizes the SST$1 \mathrm{M}$ main design parameters. The telescope achieved excellent cost $(\sim 650 \mathrm{k} € /$ telescope including manpower for massive production of telescopes) and performance ratio by adopting a well proven Davies-Cotton optics and a lightweight and compact structure of 8

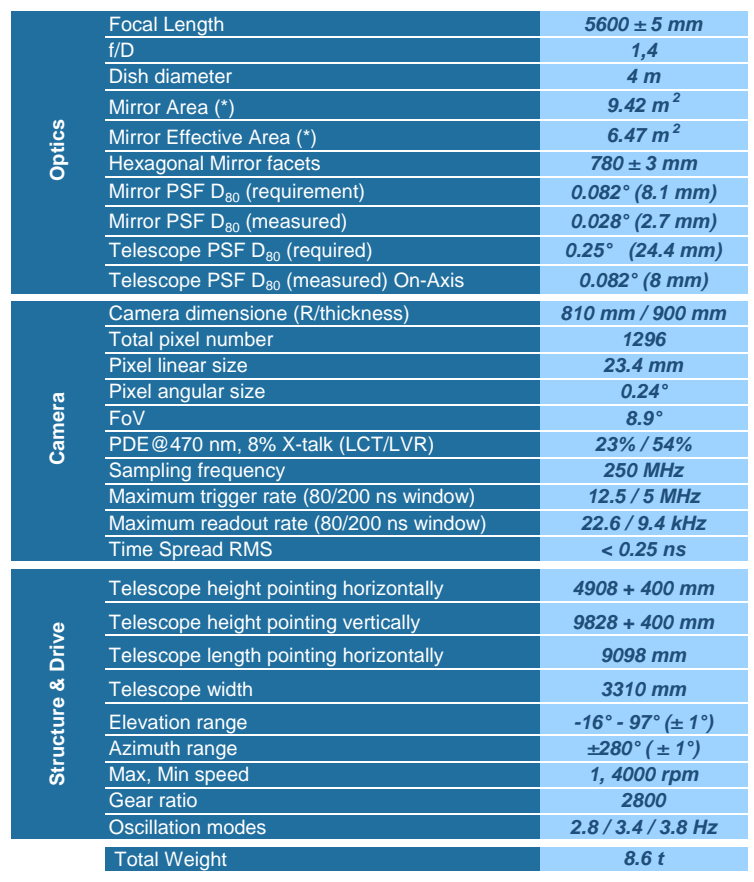

Figure 1: Main parameters of the SST-1M. Mirror effective area includes the reflectivity of the mirror and shadowing of structure.

tons designed for operations in harsh environment with minimal maintenance. The structure holds a highly performing SiPM-based camera[1], with pixel opening of about $0.24^{\circ}$ and FoV of $9^{\circ}$, achieving required sensitivity all over it. The fully digital camera delivers the waveform from each pixel in digital format with negligible deadtime fraction $\left(<10^{-7}\right)$ for random rates well beyond $1 \mathrm{kHz}$ (up to $5 \mathrm{kHz}$ in $200 \mathrm{~ns}$ readout windows). The fully digital trigger, based on FPGAs, ensures an extremely flexible system, capable of providing different triggering schemes together with dynamic and per-pixel thresholds. The camera has been fully tested and validated. Simulations show that it can reach an energy threshold of about $250 \mathrm{GeV}$ and achieve the required CTA sensitivity. Through the operation of the prototype in Krakow over the past two years and production of the second prototype, the project has reached its maturity and has gone through a final re-design.

\section{Recent upgrades and engineering for large-scale production}

The structure: The structure of the telescope underwent some improvement with respect to what waspresented in Ref. [2] and is shown in Fig. 2.

As shown in Fig. 3 the pipes and cables have been routed inside the tower instead of going through a cable carrier mounted at the top of the tower. The water and air pipes are connected to a rotary union that prevents any cable torsion. The signal, power and optical fiber are mounted on a

\footnotetext{
* Speaker.
} 


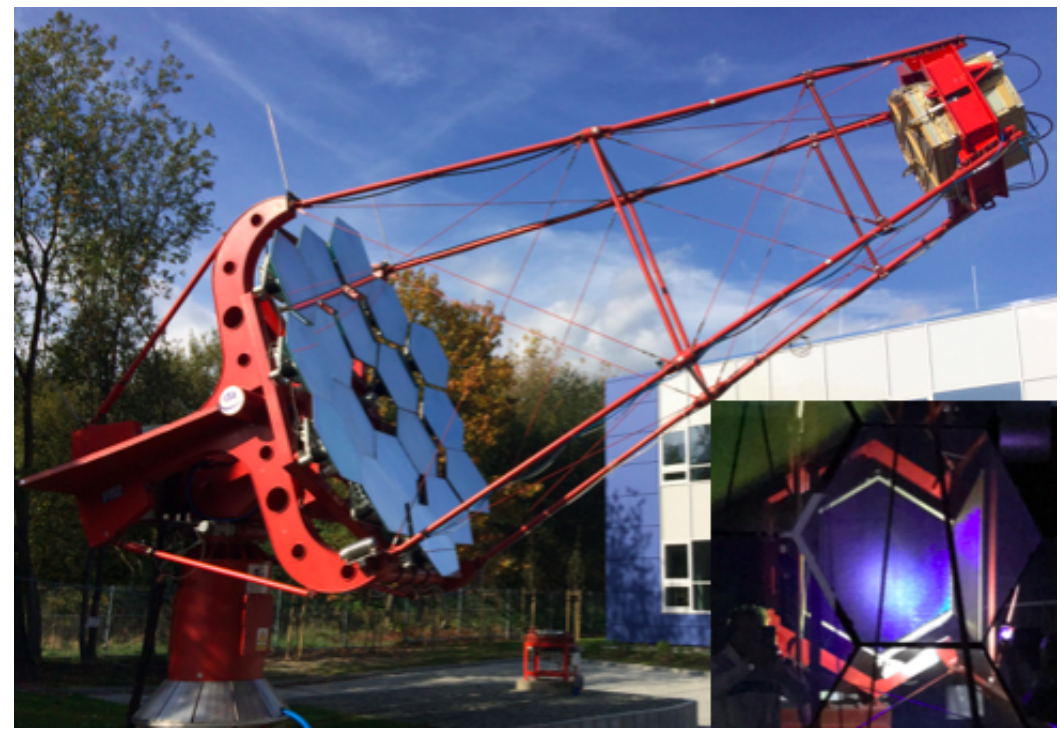

Figure 2: Image of prototype in Krakow since 2013 and camera.

sliding system which will also reduce the torsion. The pipes and cables come out of the top part of the tower and are routed to the camera through the mast using dedicated patch panels. With these modifications there is minimal exposure of cables and pipes to UV radiation, they do not contribute to the shadowing of the mirror and intervention on the telescope is safer.
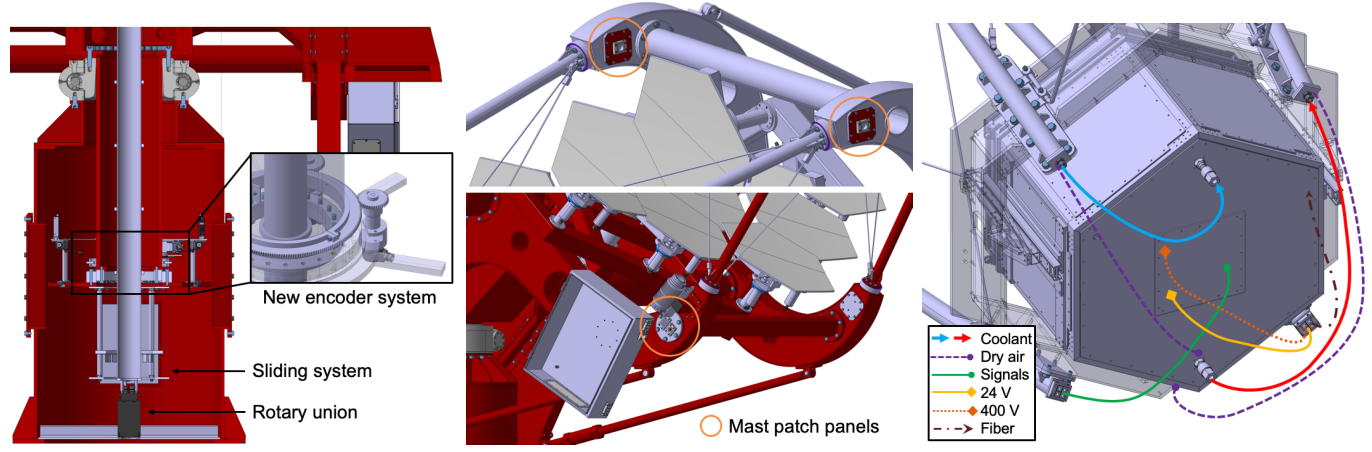

Figure 3: Left: Cut view of the improvements in the tower. Center: View of the patch panels located at the end of each mast on the dish side. Right: View of the patch panel on the camera side with the connections.

The optical system: The mirrors, i.e. substrate (S) and coating (C), will be produced entirely by Joint Lab (JL) in Olomouc for the second prototype. The first produced mirrors have demonstrated excellent performances. The maximum deviation from the nominal radius of curvature of $2 f=$ $11.2 \mathrm{~m}$ is $26.4 \mu \mathrm{m}$ for the substrate. A measurement of the point spread function (PSF) of a single hexagonal mirror facet gives a $D_{80}$ of $2.61 \mathrm{~mm}$, which is well below the CTA requirement of $8.1 \mathrm{~mm}$. These mirrors have undergone a battery of tests at the CTA mirror test facility (MTF) in Durham for validation. The procedure aims at validating the mirrors for: coating adhesion, sand blasting, impact, bird dropping resistance, temperature cycling and optical properties (reflectivity 
and single facet PSF). Except for the temperature cycling test which is still on-going, the JL mirrors have passed successfully all tests.

\subsection{PSF measurement as a function of the off-axis angle}

The optical system of the current prototype in Krakow consists of three different mirror groups from various producers. Most of the mirrors, substrate and coating, are from Galaktika ${ }^{1}$ and have been exposed to the atmospheric conditions of Krakow since the end of 2014. Another set of glass substrates from DOTI ${ }^{2}$ coated in Olomouc as well as additional full mirror sets for next telescopes. In Fig. 4 we show the prototype telescope PSF with data and simulation comparison. The agreement between the data and the simulation is shown in Fig. 5
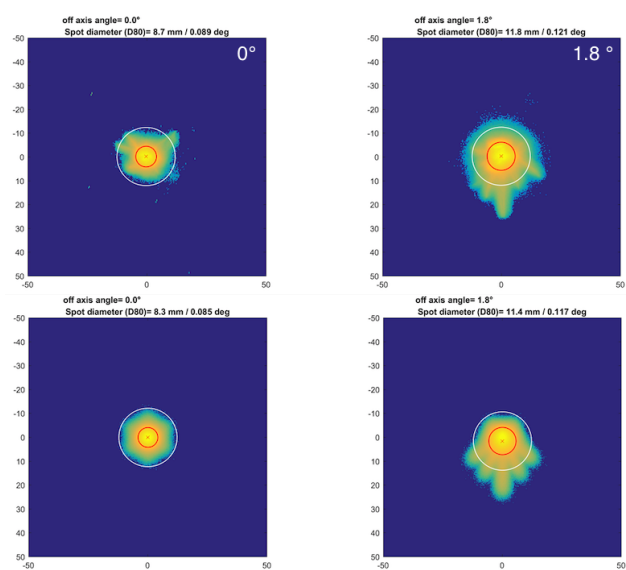
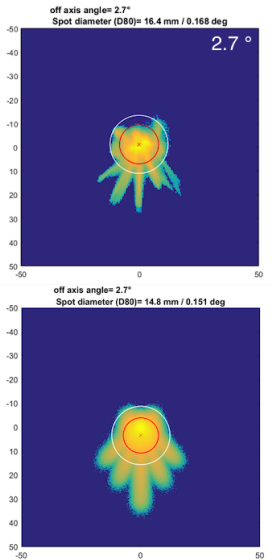
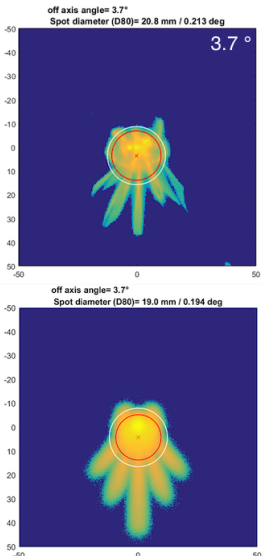

Figure 4: Upper row: measured and simulated photon images in the camera plane for different off-set angles from the optical axis. The simulation includes real reflectance and mirror surface shape of the mirrors installed on the prototype. Measured PSF is in good agreement with simulations as seen in Fig. 5.

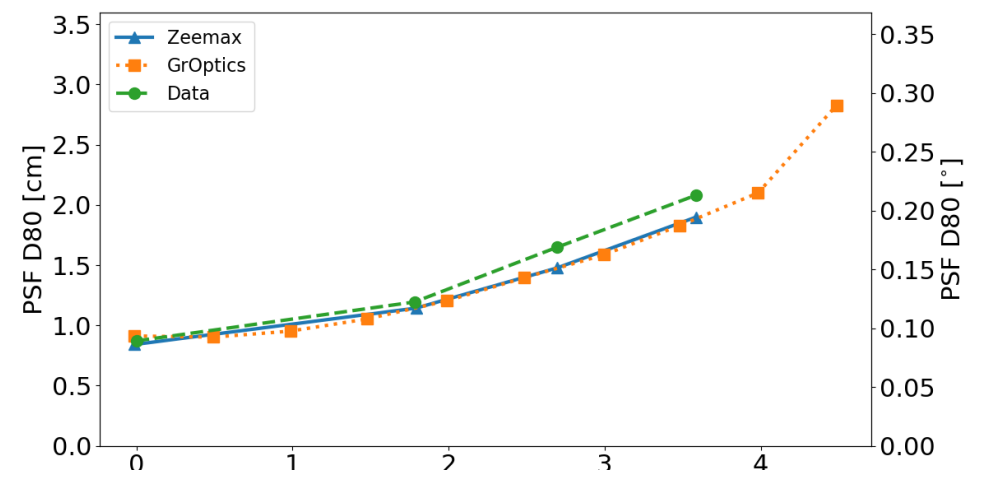

Figure 5: Comparison between measurements and simulation of the telescope PSF.

\footnotetext{
${ }^{1}$ provider of the H.E.S.S. mirrors

${ }^{2} \mathrm{US}$, provider of the VERITAS mirrors
} 


\section{The camera}

\subsection{Mechanical upgrade}

The camera mechanics have been redesigned in order to (1) facilitate the mass production process, (2) decrease the risk of water and dust ingress and (3) optimize the access for preventive and corrective maintenance operations. The first point is achieved by making all pieces machinable with CNC machines out of large aluminum blocks. Doing so, the amount of manipulations during the production process is kept to minimum. If for a single camera, the production price increases by about $20 \%$, for mass production, the gain in manpower with respect to raw material is such that a 50\% cost reduction is achieved. Fig. 6 - left shows the main blocks of the camera which have all been optimized from the first to the second prototype. This modification added to the use of Oring at all interfaces between blocks ensure the water and air tightness of the camera. Concerning the last point, the patch panel has been enlarged and the DigiCam micro crates face its opening (see Fig. 6 - right). This provide direct access to the FADC and trigger boards which combined with a revised cooling allows to remove easily individual boards and cables with minimal manual intervention. Finally, a dedicated camera holder has been designed which allows as shown in Fig. 6 - left, to slide each block of the camera onto rails and facilitate the access to both front and back of the PDP.
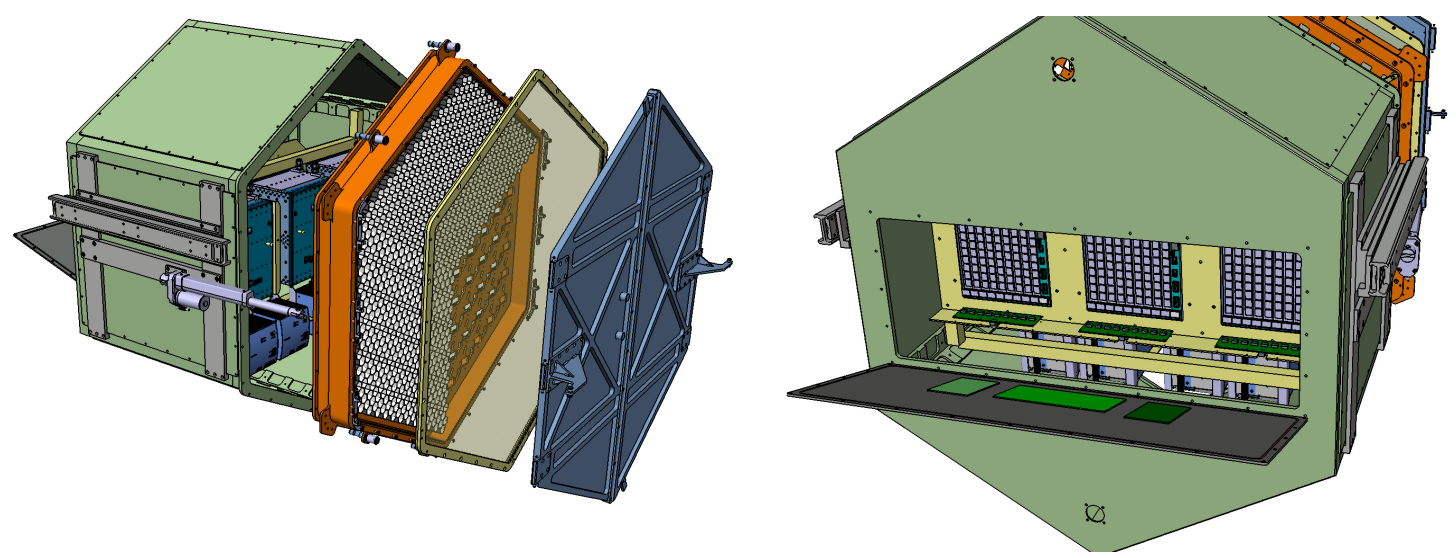

Figure 6: Left: Exploded view of the camera. The lid doors (blue), the window frame (yellow), the PDP (orange) and the camera body (green) have been optimized. Right: View from the camera patch panel which shows that one can easily access the DigiCam crates, the house keeping, LID and pointing LEDs board. In addition all the analog cables, services, power cables and optical fibers are also accessible but not depicted here.

\subsection{DigiCam firmware upgrade}

In parallel to the Cherenkov data taking, a novel approach has been taken to allow the continuous readout of the waveforms for a couple of pixels per camera with DigiCam [3]. This functionality aims at enabling the data taking for stellar intensity interferometry (SII)[4] while acquiring Cherenkov data. The challenges to achieve this readout mode are to be able to transmit the data from the FADC board to the trigger board through the backplane, then to the telescope server and finally to be able to write the data on-disk. The solution adopted is to sacrifice two trigger triplets $(8$ 
bits each) to pass the 16 bits value required for one SII channel. Doing so, the bandwidth between the FADC and trigger board is not affected and the trigger efficiency can be recovered by lowering the threshold accordingly for the cluster containing the empty triplets. The camera server and the file writing software have been modified and allow to write the $1 \mathrm{~GB} / \mathrm{s}$ sent by the camera on disk without any losses.

\subsection{Toward lager scale production}

In view of the mass production, quality assurance procedures have been defined at each stage of the production and assembly process whether they take place in institutes or industries. All procedures serve the goal of delivering a telescope which satisfies the CTA requirements. Additionally, the camera calibration strategy and validation process have been defined. Within a couple of days of data taking, the full set of calibration parameters for each pixel together with the key performance results to be confronted with the CTA requirements can be extracted. The procedure is presented in [5].

\section{Telescope control software}

SST-1M control software[6] is built around the ALMA Common Software (ACS) infrastructure originally developed for Atacama Large Millimeter Array and subsequently suggested as a standard control software framework for CTA. Each subsystem inside ACS is represented by a separate ACS component - a separate application which includes the OPC UA communication layer and all the required logic to operate the appropriate hardware. SST-1M includes the following subsystems: active mirror control, drive system, safety system, photo-detection plane, CCD cameras, DigiCam control, data acquisition system (DigiCam readout - Camera Server and raw data writer Zfitswriter), logs writer, telescope master, camera master. Observations are performed in fully automatic schedule driven mode. The schedule is created by the operator during the day and telescope master software performs all required actions including unparking and parking of the telescope, movement, tracking, changing of sources, camera control by commanding camera master, which performs dark run, trigger rate scan, data acquisition control and camera safety checks (see control software concept diagram, Fig. 7). The telescope master also implements the telescope control API developed by the Array Control And Data Acquisition (ACADA) introducing a general state machine for the whole telescope, which then is controlled by ACADA to perform all the standard operations to perform observations. To control all subsystems, also remotely, an operator is using the web-based engineering GUI. It provides means of control and monitoring for all subsystems of the telescope. During the shift an operator observes the GUI, and in case of bad weather or other issues can in real-time alter the schedule which is used by telescope master. GUI allows multiple operators or subsystem experts to operate simultaneously and perform commanding or monitoring of the telescope hardware and software. During data taking using ZeroMQ monitoring stream from Zfitswriter a randomly selected subset of events is displayed in the GUI continuously.

\section{Summary of 2018 prototype operation}

The prototype has been operated from August to December 2018. The first goal of this cam- 


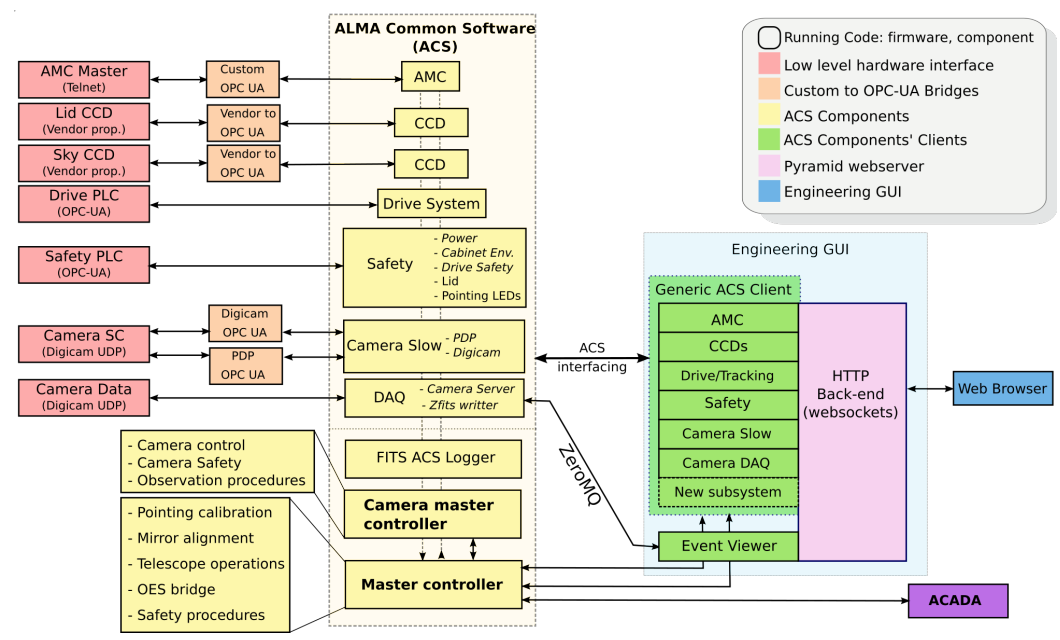

Figure 7: SST-1M control software diagram. On the left there are all the hardware components, in the middle all the ACS components responsible for each hardware component including high-level control components: telescope master and camera master. On the right there is engineering GUI (eGUI), which includes all the necessary ACS components clients.

paign was to consolidate the automation of the data taking procedure. This was achieved by the implementation and extensive test of the Master software component (see Sec. 4). All observation shifts were taken entirely remotely. This has proven that all possible operation errors could be recovered with either an automatic reaction from the system or a set of instructions handed to the shifter. Only drive faults require a local intervention which happened twice during the whole campaign due to a faulty relay which was later replaced. The observation site at IFJ, Krakow, suffers from an important light pollution due to the vicinity or a large mall complex and the town itself. Fig. 8 shows the night sky background (NSB) rate in photo electrons for the nights when the Crab nebula could be observed. It shows that most of the observation were taken with around $600 \mathrm{MHz}$ of NSB. The population above $1.2 \mathrm{GHz}$ corresponds to a night with presence of clouds. Additionally, the institute is located below the air lane of the Krakow airport. When an airplane
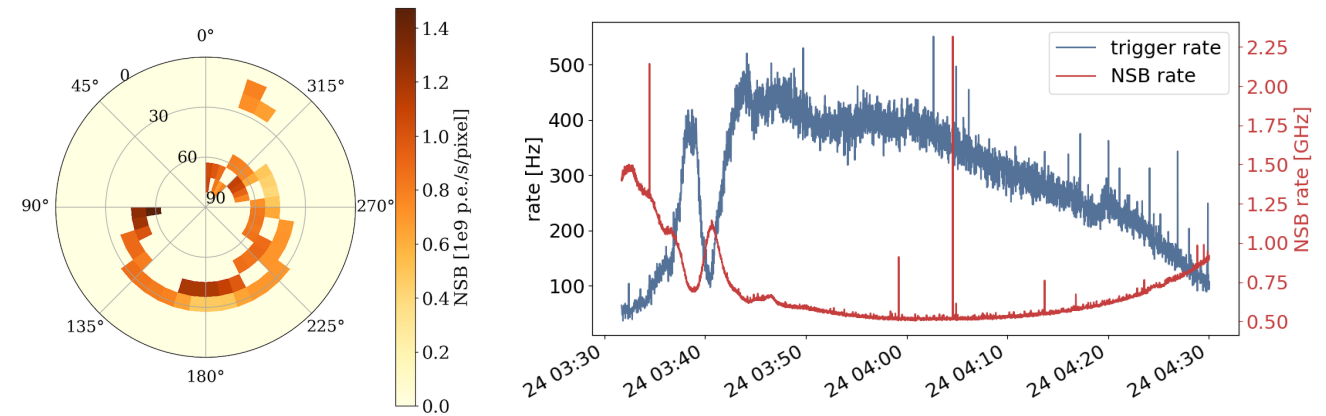

Figure 8: Left: Average NSB value as a function of azimuth and elevation angle. Right: Evolution of the NSB and trigger rate as a function of the time for a typical observation run.

passes in or close to the telescope FoV, we can observe in the camera the reflections of the city lights on its fuselage, the light which are constantly on and finally the lights that are flashing. This 
observation results in the trigger burst observed in Fig. 8-right. Each 4 ns, i.e. each sample, a trigger decision is taken based on the amplitude of the baseline subtracted signal in a group of 21 pixels. The baseline is computed for each pixel continuously as the average of the $4 \mu$ s preceding the sample under evaluation. As the planes move quickly across the FoV and emit light flashes, the baseline computed is lower than the actual light level. In this case, the pixels which have the plane in their field of view will exhibit a large signal and will cause the camera to trigger explaining the burst observed. The burst can be up to $5 \mathrm{MHz}$ which the camera can only sustain for about 40 consecutive events $(8 \mu \mathrm{s})$ given a readout window of $200 \mathrm{~ns}$. After this, the DigiCam event buffer is full and the system needs to recover. Eventually and after selecting observation runs of good quality, about $6.5 \mathrm{~h}$ of observation could be dedicated to the Crab Nebula. The preliminary results of the data analysis together with the comparison with simulated data for the Krakow site are discussed elsewhere[7].

\section{Conclusion}

The SST-1M project has used two observation campaigns and many laboratory tests to consolidate its design in order to meet the CTA requirements. It has reached the necessary maturity to plan, define and budget a reliable mass production strategy.

\section{Acknowledgement}

This work was supported by the grant Nr. DIR/WK/2017/12 from the Polish Ministry of Science and Higher Education. We greatly acknowledge financial support form the Swiss State Secretariat for Education Research and Innovation SERI. The work is supported by the projects of Ministry of Education, Youth and Sports: MEYS LM2015046, LTT17006 and EU/MEYS CZ.02.1.01/0.0/0.0/16_013/0001403, Czech Republic.

\section{References}

[1] M. Heller et al., An innovative silicon photomultiplier digitizing camera for gamma-ray astronomy, Eur. Phys. J. C77 (2017) 47 [1607.03412].

[2] Niemiec, J. and et al., Prototype of the SST-1M Telescope Structure for the Cherenkov Telescope Array, 1509.01824.

[3] Rajda, P. and et al., DigiCam - Fully digital compact read-out and trigger electronics for the SST-1M telescope proposed for the cherenkov telescope array, in Proceedings of Science, vol. 30-July-20, 2015.

[4] A. Barbano et al., Intensity Interferometry with CTA, Sept., 2018.

[5] C. Alispach et al., Calibration and operation of Si-PM based cameras for gamma-ray astronomy in presence of high night skylight, POS ( ICRC2019) 617 (2019).

[6] Sliusar, V. and et al., Control Software for the SST-1M Small-Size Telescope prototype for the Cherenkov Telescope Array, 1709.04244.

[7] J. Juryšek et al., Monte Carlo study of single SST-1M prototype for Cherenkov Telescope Array, POS ( ICRC2019) 708 (2019). 